Tema: Solidificação / Lingotamento

\title{
ESTUDO NUMÉRICO DO PROCESSO DE INERTIZAÇÃO DE DISTRIBUIDORES*
}

\author{
Bernardo Martins Braga ${ }^{1}$ \\ Roberto Parreiras Tavares ${ }^{2}$
}

\begin{abstract}
Resumo
A prática de inertização de um distribuidor de 17,7 t para lingotamento contínuo de aços foi estudada numericamente através de um modelo matemático tridimensional não-isotérmico. O modelo considera uma única fase gasosa composta de dois componentes: argônio e ar. A turbulência foi levada em conta por meio do modelo $\mathrm{k}-\varepsilon$ padrão. Os cálculos foram executados utilizando o software de CFD comercial ANSYS CFX. Diferentes configurações do sistema de inertização foram simuladas. Os resultados indicaram que a configuração atual é ineficiente e novas configurações foram sugeridas. Algumas recomendações para a correta prática de inertização também são fornecidas.
\end{abstract}

Palavras-chave: Inertização de distribuidor; Simulação matemática; Lingotamento contínuo; Inclusão não-metálica.

\section{A NUMERICAL STUDY OF THE TUNDISH INERTIZATION PRACTICE}

The inertization practice for a 17.7 tonne of steel continuous casting tundish has been studied numerically by means of a three-dimensional non-isothermal mathematical model. The model considers a single gaseous phase composed of two components: argon and air. Turbulence was accounted for with the standard k- $\varepsilon$ model. The calculations were carried out using the commercial CFD software ANSYS CFX. Different configurations of the inertization system were simulated. The results indicated that the present configuration is ineffective and new ones were suggested. Some guidelines for the correct inertization practice are also provided.

Keywords: Tundish inertization; Mathematical simulation; Continuous casting; Nonmetallic inclusion.

1 Engenheiro Metalurgista, aluno de Mestrado, programa de pós-graduação PPGEM, UFMG, bolsista do CNPq, Belo Horizonte, Minas Gerais, Brasil.

2 Engenheiro Metalurgista, PhD, Professor, Departamento de Metalurgia e Materiais, UFMG, Belo Horizonte, Minas Gerais, Brasil.

* Contribuição técnica ao $45^{\circ}$ Seminário de Aciaria - Internacional, 25 a 28 de maio de 2014, Porto Alegre, RS, Brasil. 


\section{INTRODUÇÃO}

A prática de inertização (ou purga) de distribuidor consiste na promoção de uma atmosfera inerte no interior desse reator antes da primeira corrida do sequencial. Essa técnica é amplamente utilizada para evitar perdas de produtividade e rendimento metálico decorrentes do contato entre aço e ar nas etapas transientes do processo de lingotamento contínuo: enchimento inicial e trocas de panelas.

Caso se permita o contato, o oxigênio presente no ar reage com aços acalmados produzindo inclusões não-metálicas e consumindo elementos de liga e desoxidantes como alumínio, titânio, silício e cálcio. O nitrogênio, maior constituinte do ar, também se dissolve no aço provocando a elevação ("pick-up") de seu teor [1].

Os fenômenos decorrentes da interação aço-ar podem provocar a desqualificação ou sucateamento de material metálico, sobretudo no caso de aços de alta qualidade que requerem elevada limpidez e estrito controle de composição química [2-6]. Também é favorecida a deposição de inclusões na válvula submersa, que comunica o distribuidor ao molde, propiciando sua obstrução ("clogging") [7-8].

Apesar da importância da inertização de distribuidor, há poucos trabalhos sobre o assunto disponíveis na literatura aberta. A literatura é, muitas vezes, baseada em melhorias industriais e omite informações importantes como configuração de injetores, projeto de tampa, volume do distribuidor e vazão de gás inerte. Fica evidente, portanto, a utilização massiva de soluções caseiras que são tratadas como segredo industrial.

Por outro lado, no contato com usinas siderúrgicas brasileiras e na revisão bibliográfica feita, constatou-se a presença significativa de práticas de inertização ruins em que não é feito projeto, dimensionamento e análise de eficiência. Essas práticas têm fruto na ausência de informação sobre o tema.

O dimensionamento de sistemas de inertização é normalmente baseado na teoria de reatores de mistura perfeita. Já a análise de eficiência é feita majoritariamente por abordagens experimentais como ensaios em modelos físicos e testes industriais a frio ou a quente.[6,7,9,10] Mattedi et al. [8] utilizaram um modelo matemático validado com medições industriais para projetar o sistema de inertização da CST (atual ArcelorMittal Tubarão). Entretanto os autores fornecem poucos detalhes sobre a solução de inertização desenvolvida.

Riley [7] desenvolveu importante trabalho conceitual sobre o tema. O autor considera que a perda de eficiência em sistemas de inertização ocorre pela entrada de ar oriunda da convecção natural e do entranhamento de jato turbulento. Constatou-se empiricamente que os dois mecanismos agem de forma aproximadamente aditiva. A convecção natural é atenuada por vazões altas e pelo fechamento de aberturas na tampa. Já o entranhamento é diminuído por vazões baixas, injetores em maior número e com maior diâmetro.

No presente trabalho, um modelo matemático foi utilizado para estudar o processo de inertização do distribuidor de uma usina siderúrgica nacional. A purga foi realizada pela injeção de argônio gasoso através de dutos na tampa do distribuidor. Adicionalmente, foram selecionados resultados do modelo que possibilitam orientar a correta prática de inertização e pontuar erros comuns encontrados na indústria.

\section{METODOLOGIA}

O modelo matemático utilizado resolve as equações de conservação de massa, momentum e energia (formulação para baixo número de Mach) da mistura gasosa. É

\footnotetext{
* Contribuição técnica ao 45 Seminário de Aciaria - Internacional, 25 a 28 de maio de 2014 Porto Alegre, RS, Brasil.
} 
resolvida também uma equação de conservação de massa para o ar em termos de sua fração mássica. A turbulência é modelada pelo modelo k- $\varepsilon$ padrão com a adição de termos de produção e dissipação não direcional de turbulência devido ao empuxo [11]. O software comercial ANSYS CFX foi usado nos cálculos.

Os gases são tratados como ideais. A capacidade calorífica, condutividade térmica e viscosidade do ar foram retiradas de Incropera et al.[12]. Todas as propriedades do argônio podem ser preditas teoricamente. Utilizaram-se os valores de condutividade térmica e viscosidade calculados por Bich, Millat e Vogel [13] com correções relativistas e a capacidade calorífica de gás ideal monoatômico [14]

A difusividade entre ar e argônio, a condutividade térmica da mistura e a viscosidade da mistura foram calculadas segundo Bird, Stewart e Lighfoot [15]. A dependência de todas as propriedades dos materiais com a temperatura foi considerada por expressões ou através de um esquema de inversão de tabelas. A Figura 1 apresenta um esquema do distribuidor modelado. Devido à simetria do sistema, apenas metade do domínio foi simulada.

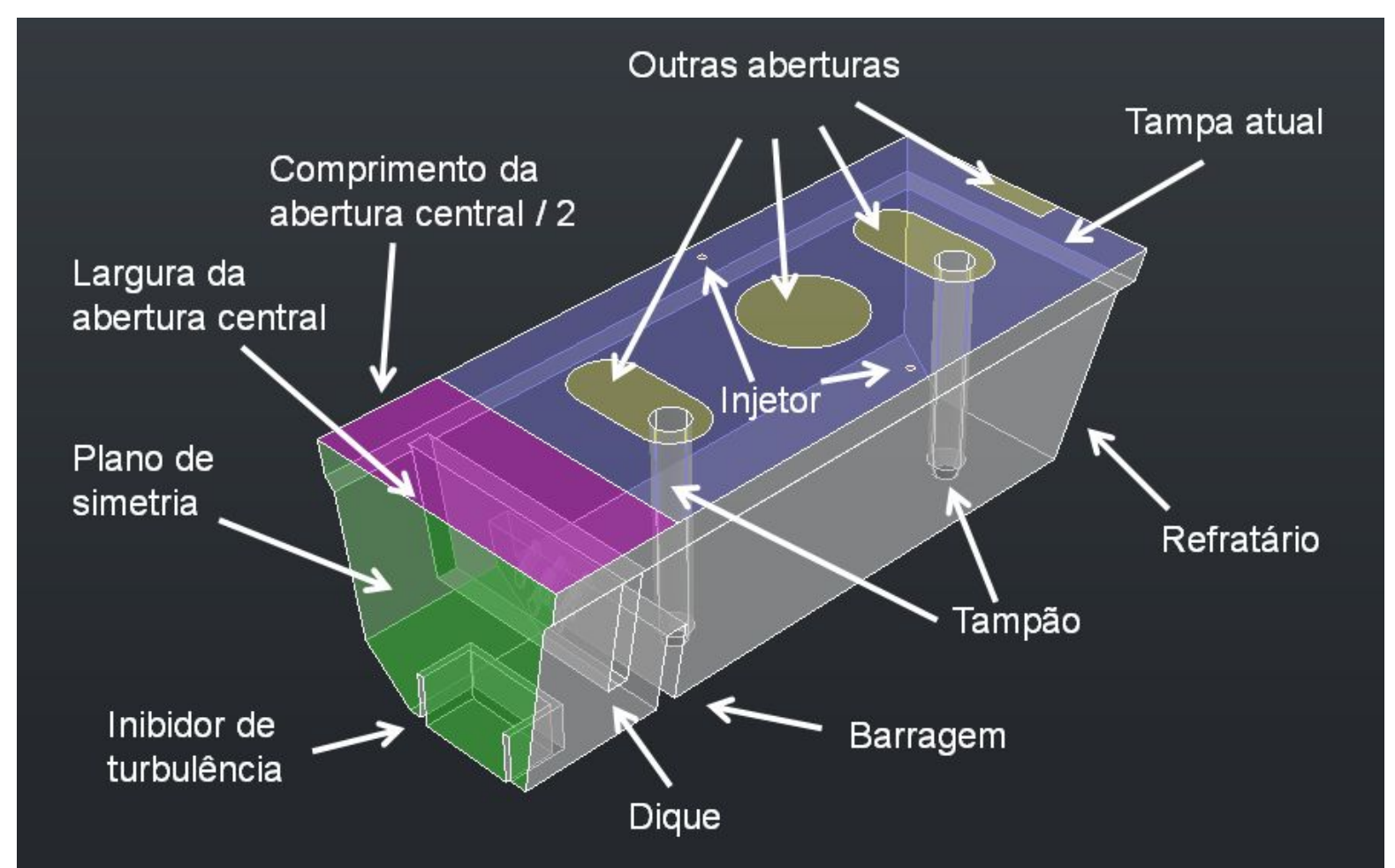

Figura 1. Esquema ilustrativo de distribuidor com injeção de argônio gasoso por 4 dutos de $25.4 \mathrm{~mm}$ na tampa (casos F1, F4 e Q1, ver Tabelas 1 e 2). Devido à simetria, apenas metade do domínio foi simulada.

Com objetivo de estudar separadamente os dois mecanismos de entrada de ar e de tornar os resultados de aplicação mais geral, foram simulados casos de inertização em distribuidor com partida a frio (Tabela 1) e com partida a quente (Tabela 2). No primeiro caso, assumiu-se temperatura inicial do ar presente no distribuidor e dos refratários de $25^{\circ} \mathrm{C}$. No segundo caso, foi utilizada temperatura de $1200^{\circ} \mathrm{C}$.

\footnotetext{
* Contribuição técnica ao 45 Seminário de Aciaria - Internacional, 25 a 28 de maio de 2014, Porto Alegre, RS, Brasil.
} 
Tabela 1. Casos estudados de inertização em distribuidor com partida a frio

\begin{tabular}{|l|l|l|l|l|l|l|l|}
\hline Caso & F1 & F2 & F3 & F4 & F5 & F6 & F7 \\
\hline Vazão total $\left(\mathrm{Nm}^{3} / \mathrm{h}\right)$ & 70 & 70 & 70 & 560 & 70 & 105 & 140 \\
\hline $\mathrm{N}^{\circ}$ de injetores & 4 & 12 & 4 & 4 & 4 & 4 & 4 \\
\hline $\begin{array}{l}\text { Diâmetro dos } \\
\text { injetores (mm) }\end{array}$ & 25,4 & 25,4 & 76,2 & 25,4 & 25,4 & 25,4 & 25,4 \\
\hline $\begin{array}{l}\text { Largura abertura } \\
\text { central (mm) }\end{array}$ & 1038,20 & 1038,20 & 1038,20 & 1038,20 & 920,68 & 920,68 & 920,68 \\
\hline $\begin{array}{l}\text { Comprimento da } \\
\text { abertura central (mm) }\end{array}$ & 920 & 920 & 920 & 920 & 596 & 596 & 596 \\
\hline Outras aberturas & Abertas & Abertas & Abertas & Abertas & Fechadas & Fechadas & Fechadas \\
\hline
\end{tabular}

Tabela 2. Casos estudados de inertização em distribuidor com partida a quente

\begin{tabular}{|l|l|l|l|l|}
\hline Caso & Q1 & Q2 & Q3 & Q4 \\
\hline Vazão total $\left(\mathrm{Nm}^{3} / \mathrm{h}\right)$ & 70 & 70 & 105 & 140 \\
\hline $\mathrm{N}^{\circ}$ de injetores & 4 & 4 & 4 & 4 \\
\hline $\begin{array}{l}\text { Diâmetro dos } \\
\text { injetores (mm) }\end{array}$ & 25,4 & 25,4 & 25,4 & 25,4 \\
\hline $\begin{array}{l}\text { Largura abertura } \\
\text { central (mm) }\end{array}$ & 1038,20 & 920,68 & 920,68 & 920,68 \\
\hline $\begin{array}{l}\text { Comprimento da } \\
\text { abertura central } \\
\text { (mm) }\end{array}$ & 920 & 596 & 596 & 596 \\
\hline \begin{tabular}{l} 
Outras aberturas \\
\hline
\end{tabular} & Abertas & Fechadas & Fechadas & Fechadas \\
\hline
\end{tabular}

A fim de se discutir práticas industriais encontradas, o caso F1 também foi simulado na ausência de tampa ( $F 1 \mathrm{sem})$ e com os injetores reposicionados nas aberturas circulares da tampa (F1circ).

As principais características do distribuidor, as condições de contorno usadas e a condição inicial imposta ao modelo são apresentadas, respectivamente, nas Tabelas 3, 4 e 5. Assumiu-se nos cálculos pressão ambiente de $1 \mathrm{~atm}$.

Tabela 3. Características principais do distribuidor em estudo

\begin{tabular}{|l|l|l|l|}
\hline Capacidade nominal $(\mathrm{t})$ & 17,7 & $\begin{array}{l}\text { Área aberta na tampa }\left(\mathrm{m}^{2}\right), \\
\text { casos F1 a F4 e Q1 }\end{array}$ & 1,70 \\
\hline Volume interno $\left(\mathrm{m}^{3}\right)$ & 3,20 & $\begin{array}{l}\text { Área aberta na tampa }\left(\mathrm{m}^{2}\right), \\
\text { casos F5 a F7, Q2 e Q3 }\end{array}$ & 0,55 \\
\hline
\end{tabular}

* Contribuição técnica ao 450 Seminário de Aciaria - Internacional, 25 a 28 de maio de 2014, Porto Alegre, RS, Brasil. 
Tabela 4. Condições de contorno utilizadas no modelo matemático

\begin{tabular}{|c|c|c|c|c|}
\hline \multicolumn{2}{|l|}{ Entrada } & \multicolumn{2}{|c|}{ Aberturas* } & \multirow{2}{*}{$\begin{array}{c}\text { Refratários e tampa } \\
\text { Temperatura constante }\end{array}$} \\
\hline Perfil & Uniforme & $\begin{array}{l}\text { Pressão relativa } \\
(\mathrm{Pa})\end{array}$ & 0 & \\
\hline $\begin{array}{l}\text { Distribuição de } \\
\text { gás entre } \\
\text { injetores }\end{array}$ & Uniforme & Direção & Normal & Não escorregamento \\
\hline Temperatura $\left({ }^{\circ} \mathrm{C}\right)$ & 25 & Temperatura $\left({ }^{\circ} \mathrm{C}\right)$ & 25 & Parede hidraulicamente lisa \\
\hline 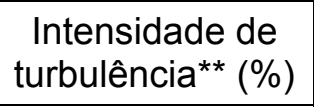 & 5 & 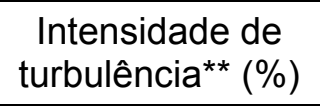 & 1 & $\begin{array}{c}\text { Função de parede } \\
\text { escalável }\end{array}$ \\
\hline $\begin{array}{c}\text { Razão de } \\
\text { viscosidade }^{\star * *}\end{array}$ & 10 & $\begin{array}{c}\text { Razão de } \\
\text { viscosidade*** }\end{array}$ & 1 & Simetria \\
\hline Fração de ar & 0 & Fração de ar & 1 & Fluxo zero \\
\hline
\end{tabular}

* A pressão total relativa é utilizada para entrada de gás e a pressão estática relativa para saída de gás. Os demais parâmetros só são usados caso haja entrada de gás [11].

** Relação (I) entre a componente flutuante da velocidade do fluido (u), presente em escoamentos turbulentos, e a velocidade média do fluido (U): I = u / U.[11].

*** Razão $(R)$ entre a viscosidade turbulenta $\left(\mu_{t}\right)$, introduzida pela hipótese Boussinesq, e a viscosidade dinâmica do material $(\mu): R=\mu_{t} / \mu[11]$.

Tabela 5. Condição inicial imposta ao modelo matemático

\begin{tabular}{|c|c|c|c|}
\hline $\begin{array}{c}\text { Velocidades no } \\
\text { interior do } \\
\text { domínio }\end{array}$ & Nula & $\begin{array}{c}\text { Intensidade de } \\
\text { turbulência (\%) }\end{array}$ & 1 \\
\hline $\begin{array}{c}\text { Pressão no } \\
\text { interior do } \\
\text { domínio }\end{array}$ & Hidrostática & $\begin{array}{c}\text { Razão de } \\
\text { viscosidade }\end{array}$ & 1 \\
\hline $\begin{array}{c}\text { Temperatura no } \\
\text { interior do } \\
\text { domínio }\end{array}$ & Uniforme & Fração de ar & 1 \\
\hline
\end{tabular}

A Figura 2 apresenta as distribuições de injetores na tampa do distribuidor utilizadas nesse trabalho.

O modelo matemático prevê a evolução no tempo da fração molar de argônio e da temperatura em cada ponto do distribuidor e seus respectivos valores médios. As Figuras 3 e 4 mostram exemplos de resultados típicos. As oscilações nas curvas da Figura 4 decorrem da variação no tempo da entrada de ar no distribuidor como pode ser constatado na Figura 3. Essa periodicidade é motivada pela convecção natural.

* Contribuição técnica ao 450 Seminário de Aciaria - Internacional, 25 a 28 de maio de 2014, Porto Alegre, RS, Brasil. 


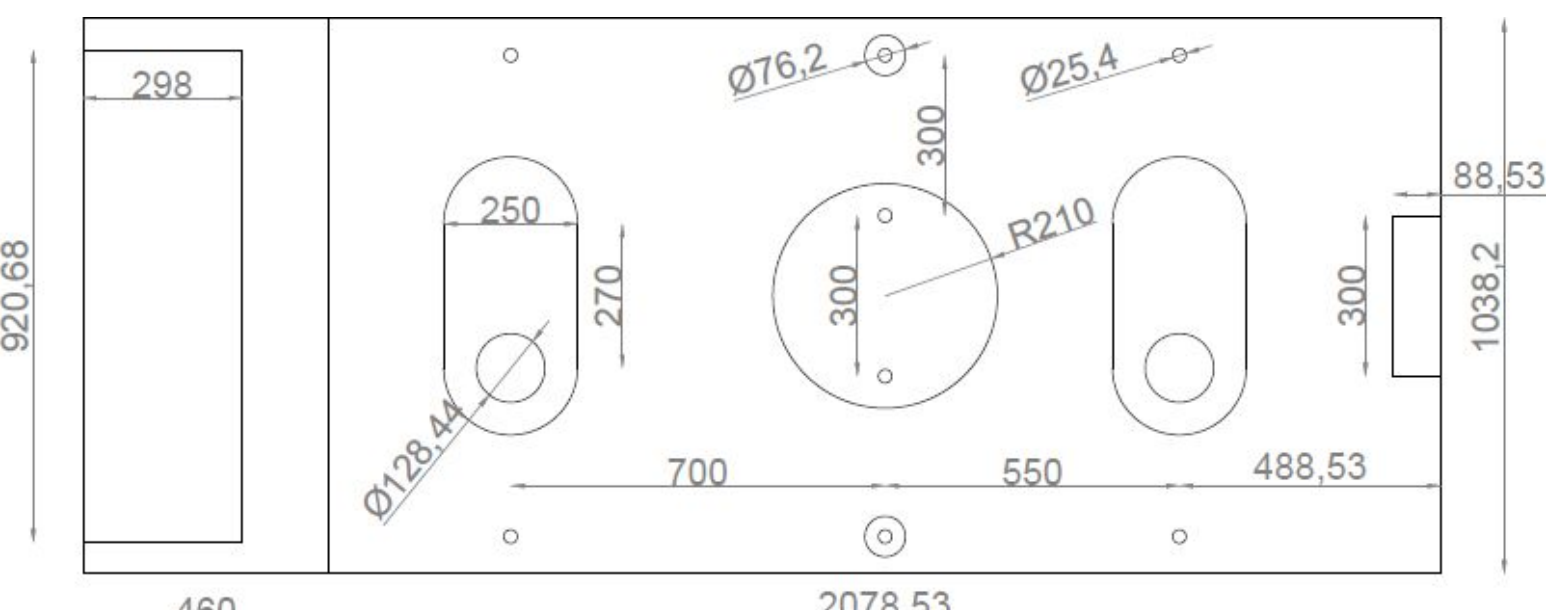

460

2078,53

Figura 2. Esquema da tampa do distribuidor utilizada nesse trabalho com todas as configurações de abertura central e de injetores sobrepostas. Nos casos que utilizam 4 injetores, apenas os dutos centrais são modelados. Os dutos localizados na abertura circular só são usados no caso F1circ. Dimensões em milímetros.

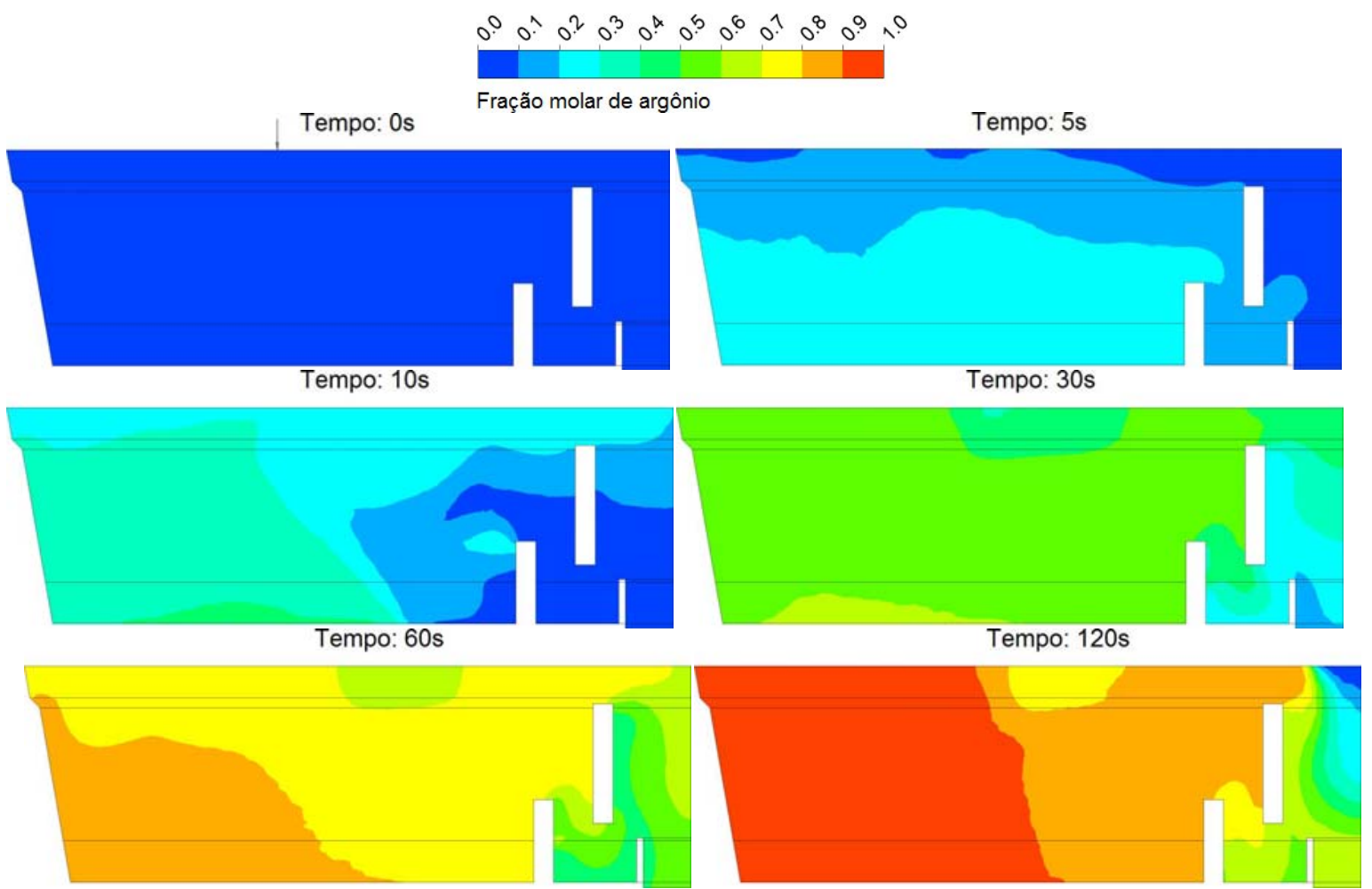

Figura 3. Contornos da fração molar de argônio prevista pelo modelo matemático para o caso Q2 (ver tabela 2). Os contornos foram construídos no plano central do distribuidor. A seta na primeira figura indica o ponto de injeção de argônio.

No caso de partida a frio, a simulação foi executada por 5 minutos - tempo máximo disponível para a purga na indústria. Já para partida a quente, o teor de argônio se estabiliza mais rapidamente e simulou-se apenas 2,5 minutos.

* Contribuição técnica ao 45 Seminário de Aciaria - Internacional, 25 a 28 de maio de 2014, Porto Alegre, RS, Brasil. 

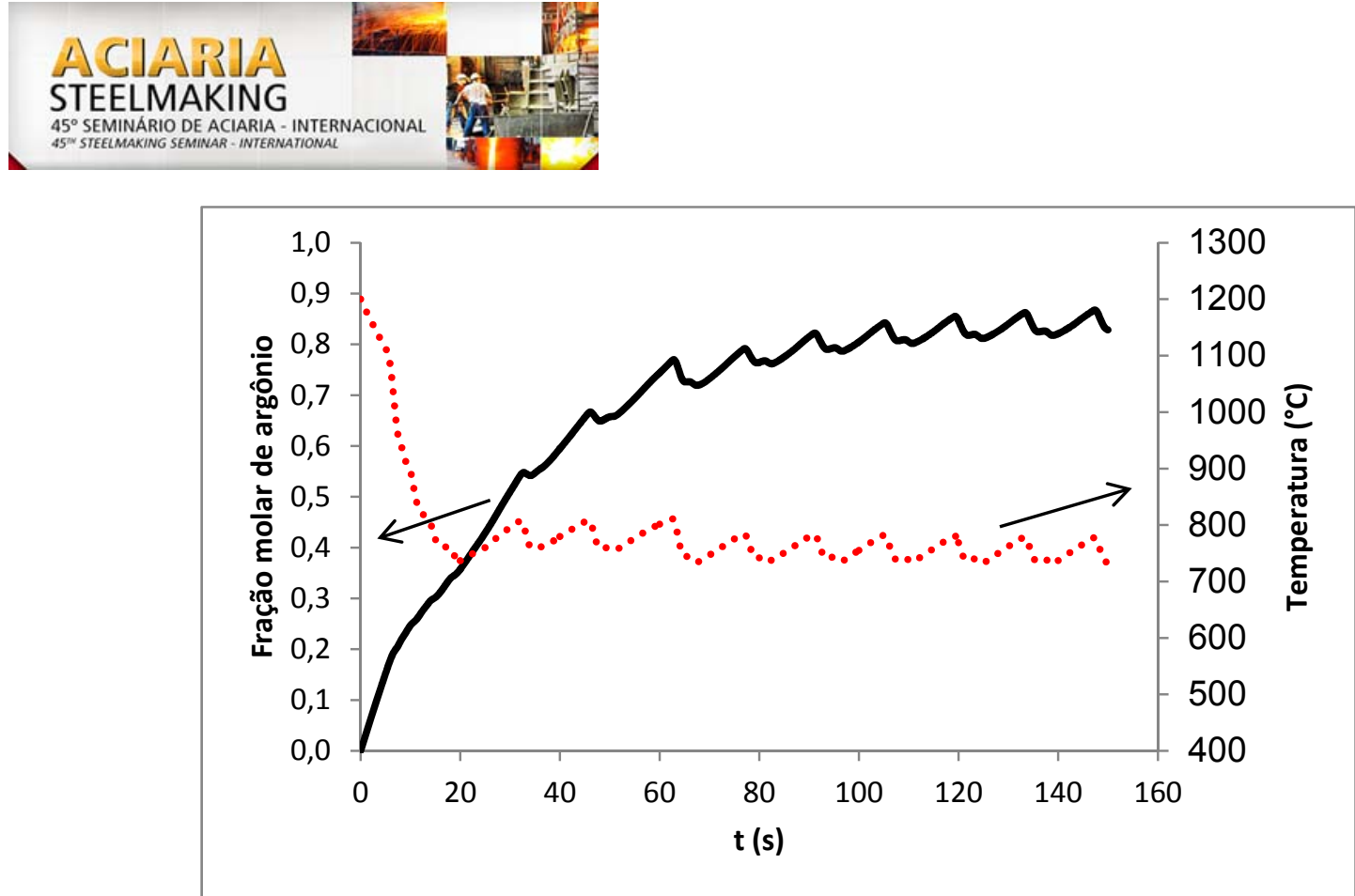

Figura 4. Variação no tempo da fração molar de argônio e da temperatura para caso Q2. Os valores representam médias volumétricas no interior do distribuidor.

Para garantir a acurácia numérica do modelo, foram realizados estudos de independência de malha e de passo de tempo. As simulações finais utilizaram malhas tetraédricas de aproximadamente 330 mil elementos (80 mil nós) e passo de tempo de $0,1 \mathrm{~s}$.

Para efeito de comparação, a eficiência de purga calculada pela teoria de reatores de mistura perfeita também é apresentada. Nesse caso, foi desconsiderada a entrada de ar devido à dificuldade em se estimar seu valor a priori. A equação pertinente é [7-9]:

$$
\mathrm{N}_{\mathrm{Ar}}=1-\exp \left(-\frac{\mathrm{Q}_{\mathrm{CNTP}}}{\mathrm{V}_{\text {dist }}} \frac{\mathrm{T}_{\text {dist }}}{\mathrm{T}_{\mathrm{CNTP}}} \mathrm{t}\right)
$$

Em que $\mathrm{N}_{\mathrm{Ar}}$ é a fração molar (numericamente igual a fração volumétrica) de argônio na fase gasosa, Q QNTP a vazão de argônio $\left(\mathrm{Nm}^{3} / \mathrm{min}\right)$ medida na CNTP, T CNTP a temperatura da CNTP $(\mathrm{K}), \mathrm{V}_{\text {dist }} \mathrm{o}$ volume do distribuidor $\left(\mathrm{m}^{3}\right), \mathrm{T}_{\text {dist }}$ a temperatura no interior do distribuidor $(\mathrm{K})$ e $\mathrm{t}$ o tempo (min). Nesse trabalho, foi adotada a convenção antiga de CNTP: 1atm e 273,15K. A Equação 1 assume que o gás se comporta idealmente.

Os resultados da equação de mistura perfeita serão apresentados na forma MPXXX, em que $X X X$ é a vazão em Nm $3 / h$. Por exemplo, MP070 representa o valor da fração molar de argônio predita pela equação para vazão de $70 \mathrm{Nm}^{3} / \mathrm{h}$. A temperatura do distribuidor usada nos cálculos foi $25^{\circ} \mathrm{C}$ para partida a frio. Já para partida a quente, utilizou-se tanto a temperatura média final prevista pelo modelo matemático quanto à temperatura de pré-aquecimento dos refratários $\left(1200^{\circ} \mathrm{C}\right)$.

\section{RESULTADOS E DISCUSSÃO}

A Tabela 6 apresenta a fração molar média de argônio $\left(\mathrm{N}_{\mathrm{Ar}}\right)$ no interior do distribuidor ao fim da purga predita pelo modelo matemático e pela equação de mistura perfeita para os casos estudados de inertização de distribuidor com partida a frio.

* Contribuição técnica ao 45 Seminário de Aciaria - Internacional, 25 a 28 de maio de 2014, Porto Alegre, RS, Brasil. 
Tabela 6. Fração molar média de argônio $\left(\mathrm{N}_{\mathrm{Ar}}\right)$ no interior do distribuidor ao fim da purga predita pelo modelo matemático e pela equação de mistura perfeita para casos estudados de inertização de distribuidor com partida a frio

\begin{tabular}{|l|l|l|l|l|l|l|l|}
\hline Caso & $\mathrm{N}_{\mathrm{Ar}}$ & Caso & $\mathrm{N}_{\mathrm{Ar}}$ & Caso & $\mathrm{N}_{\mathrm{Ar}}$ & Caso & $\mathrm{N}_{\mathrm{Ar}}$ \\
\hline F1 & 0,72 & $\mathrm{~F} 2$ & 0,85 & $\mathrm{~F} 5$ & 0,89 & MP070 & 0,86 \\
\hline F1 sem & 0,12 & $\mathrm{~F} 3$ & 0,90 & $\mathrm{~F} 6$ & 0,97 & MP105 & 0,95 \\
\hline F1 circ & 0,20 & F4 & 0,33 & F7 & 0,99 & MP140 & 0,98 \\
\hline
\end{tabular}

$\mathrm{O}$ caso $\mathrm{F} 1$ corresponde à versão de partida a frio da configuração atual utilizada na usina (Q1). A fração molar de argônio predita $\left(\mathrm{N}_{\mathrm{Ar}}=0,72\right)$ indica uma eficiência de purga razoável. Os casos $\mathrm{F} 2\left(\mathrm{~N}_{\mathrm{Ar}}=0,85\right)$ e $\mathrm{F} 3\left(\mathrm{~N}_{\mathrm{Ar}}=0,90\right)$ mostram que a eficiência de purga pode ser melhorada ao se utilizar injetores em maior número ou com maior diâmetro. Esse fato decorre da diminuição de entrada de ar por entranhamento de jato turbulento, conforme Riley [7].

O entranhamento também pode ser reduzido pela proteção física do jato de argônio em relação ao ar atmosférico. Essa ideia foi utilizada no caso $5\left(\mathrm{~N}_{\mathrm{Ar}}=0,89\right)$, em que se fechou aberturas na tampa do distribuidor. Embora a proteção do jato resolva o problema de entrada de ar do caso F1, pode ser interessante usar em conjunto configurações de injetores menos propícias ao arraste de ar, como em F2 e F3, para tornar o processo robusto em relação a uma eventual má selagem das aberturas na tampa.

A tentativa de se aumentar a eficiência de purga do caso $\mathrm{F} 1$ pelo aumento excessivo da vazão de argônio $\left(70 \mathrm{Nm}^{3} / \mathrm{h}\right.$ para $\left.560 \mathrm{Nm}^{3} / \mathrm{h}\right)$ corresponde ao caso $\mathrm{F} 4$. Constatase, no entanto, que a fração de argônio cai significativamente $\left(\mathrm{N}_{\mathrm{Ar}}=0,33\right)$. Esse resultado é explicado pelo entranhamento de jato turbulento, pois a quantidade de ar capturada do entorno depende da vazão de argônio utilizada [7].

O resultado anterior alerta para o risco de uma prática comum: utilizar a vazão máxima disponível na linha. Ele também aponta que a inertização de distribuidores maiores é mais delicada, já que nesse caso se lida inerentemente com vazões altas, inclusive maiores que do caso F4.

A inertização em distribuidor sem tampa, $\mathrm{F} 1 \mathrm{sem}\left(\mathrm{N}_{\mathrm{Ar}}=0,12\right)$, ou por injetores localizados nas aberturas da tampa, $\mathrm{F} 1$ circ $\left(\mathrm{N}_{\mathrm{Ar}}=0,20\right)$, são práticas encontradas na indústria brasileira. Esses procedimentos provocam severo arraste de ar para dentro do distribuidor e eficiências de purga muito baixas. Esse resultado também serve de alerta para o risco de se injetar argônio por dutos na abertura central como uma tentativa de se reduzir a maior concentração de ar encontrada nessa região (Figura 3).

Os casos F6 $\left(\mathrm{N}_{\mathrm{Ar}}=0,97\right)$ e $\mathrm{F} 7\left(\mathrm{~N}_{\mathrm{Ar}}=0,99\right)$ mostram que, com o controle adequado da entrada de ar, é possível aumentar a eficiência de purga com o aumento de vazão. Embora a vazão de $105 \mathrm{Nm}^{3} / \mathrm{h}$ (caso $\mathrm{F} 6$ ) seja suficiente para obter teor de oxigênio residual inferior a $1 \%\left(\mathrm{~N}_{\mathrm{Ar}}<0,05\right)$, a vazão de $140 \mathrm{Nm}^{3} / \mathrm{h}$ (caso $\mathrm{F} 7$ ) é recomendada por permitir alcançar o mesmo resultado com alguma folga de tempo.

Vale ressaltar aqui que mesmo a vazão máxima disponível na linha pode não ser suficiente para inertizar completamente o distribuidor. Assim é altamente recomendado o uso de medidores de vazão calibrados em sistemas de inertização, o que não é regra na prática industrial.

* Contribuição técnica ao 45 Seminário de Aciaria - Internacional, 25 a 28 de maio de 2014, Porto Alegre, RS, Brasil. 
Embora apresentem valores de $\mathrm{N}_{\mathrm{Ar}}$ ligeiramente inferiores, as previsões do modelo de mistura perfeita concordam com aquelas feitas pelo modelo matemático, desde que a entrada de ar seja eliminada.

As Tabelas 7 e 8 apresentam os resultados obtidos, respectivamente, pelo modelo matemático e pela equação de mistura perfeita para a inertização de distribuidor com partida a quente.

Tabela 7. Fração molar média de argônio $\left(\mathrm{N}_{\mathrm{Ar}}\right)$ e temperatura média $(T)$ no interior do distribuidor ao fim da purga predita pelo modelo matemático para casos estudados de inertização de distribuidor com partida a quente

\begin{tabular}{|l|l|l|l|l|l|}
\hline Caso & $\mathrm{T}\left({ }^{\circ} \mathrm{C}\right)$ & $\mathrm{N}_{\mathrm{Ar}}$ & $\mathrm{Caso}$ & $\mathrm{T}\left({ }^{\circ} \mathrm{C}\right)$ & $\mathrm{N}_{\mathrm{Ar}}$ \\
\hline $\mathrm{Q} 1$ & 198 & 0,06 & $\mathrm{Q} 3$ & 732 & 0,90 \\
\hline Q2 & 733 & 0,83 & $\mathrm{Q} 4$ & 750 & 1,00 \\
\hline
\end{tabular}

Tabela 8. Fração molar média de argônio $\left(\mathrm{N}_{\mathrm{Ar}}\right)$ no interior do distribuidor ao fim da purga predita pela equação de mistura perfeita para casos estudados de inertização de distribuidor com partida a quente. Também é fornecida a temperatura do distribuidor $(T)$ considerada nos cálculos

\begin{tabular}{|l|l|l|l|l|l|}
\hline Caso & $\mathrm{T}\left({ }^{\circ} \mathrm{C}\right)$ & $\mathrm{N}_{\mathrm{Ar}}$ & Caso & $\mathrm{T}\left({ }^{\circ} \mathrm{C}\right)$ & $\mathrm{N}_{\mathrm{Ar}}$ \\
\hline MP070 & 733 & 0,97 & MP070 & 1200 & 0,99 \\
\hline MP105 & 732 & 0,99 & MP105 & 1200 & 1,00 \\
\hline MP140 & 750 & 1,00 & MP140 & 1200 & 1,00 \\
\hline
\end{tabular}

O caso Q1 $\left(\mathrm{N}_{\mathrm{Ar}}=0,06\right)$, que corresponde à prática atual na usina, possui eficiência de purga extremamente baixa e muito inferior ao caso $\mathrm{F} 1$, devido à entrada de ar adicional por convecção natural. Fica claro que o projeto de sistemas de inertização para distribuidores com partida a quente é mais desafiador que para distribuidores com partida a frio.

O caso Q2 $\left(\mathrm{N}_{\mathrm{Ar}}=0,83\right)$ mostra que o fechamento de aberturas na tampa é capaz de elevar substancialmente a eficiência de purga em relação ao caso Q1. Essa melhoria deve-se ao combate simultâneo dos dois mecanismos de entrada de ar.

Assim como no caso de distribuidor com partida a frio, a eficiência de purga pode ser aumentada em distribuidor com partida a quente pelo aumento de vazão. Nesse último, o efeito benéfico é potencializado, pois o aumento de vazão reduz também a entrada de ar por convecção natural [7] Os casos Q3 $\left(N_{A r}=0,90\right)$ e Q4 $\left(N_{A r}=1,00\right)$, que são análogos aos casos $\mathrm{F} 6$ e $\mathrm{F} 7$, demonstram isso. A vazão de $140 \mathrm{Nm}^{3} / \mathrm{h}$ (caso Q4) é recomendada.

Interessante notar que a eficiência de purga do caso Q4 $\left(\mathrm{N}_{\mathrm{Ar}}=1,00\right)$ é superior à do caso $\mathrm{F} 7\left(\mathrm{~N}_{\mathrm{Ar}}=0,99\right)$. Esse resultado é explicado pela expansão do argônio que ocorre em distribuidor com partida a quente. De fato, a vazão volumétrica efetiva nessa situação é superior ao caso com partida a frio. A equação (1) também incorpora esse efeito. Tal comportamento só é notado na ausência de entrada de ar. Novamente o modelo de mistura perfeita prevê adequadamente a eficiência de purga, desde que seja eliminada a entrada de ar - caso Q4. No presente estudo de

* Contribuição técnica ao 450 Seminário de Aciaria - Internacional, 25 a 28 de maio de 2014, Porto Alegre, RS, Brasil. 


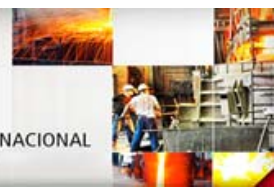

caso, o desconhecimento da temperatura do gás no interior do distribuidor não foi um problema para a aplicação da equação, pois ela é pouco sensível à variações de temperatura quando $\mathrm{N}_{\mathrm{Ar}}$ é próximo da unidade.

\section{CONCLUSÕES}

Através do uso de um modelo matemático, foi desenvolvido um estudo de caso em que se buscaram configurações adequadas para o sistema de inertização do distribuidor de uma usina siderúrgica nacional. Adicionalmente, procurou-se pontuar os erros comuns encontrados na indústria. Desse trabalho, conclui-se que:

- O modelo matemático desenvolvido realiza predições coerentes com a literatura;

- A equação de mistura perfeita apresenta resultados condizentes com o modelo matemático, desde que não haja entrada de ar;

- A configuração de inertização atual resulta em eficiência de purga razoável $\left(\mathrm{N}_{\mathrm{Ar}}=0,72\right)$ no caso de partida a frio do distribuidor e eficiência extremamente baixa para partida a quente $\left(\mathrm{N}_{\mathrm{Ar}}=0,06\right)$;

- O uso de injetores em maior número ou com maior diâmetro e a proteção física do jato são eficazes em diminuir a entrada de ar no distribuidor por entranhamento de jato turbulento;

- Aumento da vazão de argônio pode resultar em menor eficiência de purga, caso não se atente para a entrada de ar;

- A inertização de distribuidores de grande capacidade é delicada devido às altas vazões de argônio inerentemente utilizadas;

- As práticas de injeção de argônio em distribuidor sem tampa por dutos em sua superfície e injeção de argônio por dutos nas aberturas da tampa são extremamente ineficientes devido à significativa entrada de ar;

- A purga de distribuidores com partida a quente é mais desafiadora que para distribuidores com partida a frio devido à entrada de ar adicional por convecção natural;

- O fechamento de aberturas na tampa é eficiente em reduzir a entrada de ar por convecção natural;

- A vazão efetiva em distribuidor com partida a quente é superior à do caso com partida a frio, entretanto esse efeito pode ser mascarado pela entrada de ar;

- Para o caso em estudo, a prática de inertização recomendada tanto para partida a frio $\left(\mathrm{N}_{\mathrm{Ar}}=0,99\right)$ quanto a quente $\left(\mathrm{N}_{\mathrm{Ar}}=1,00\right)$ é vazão de $140 \mathrm{Nm}^{3} / \mathrm{h}$ com fechamento de aberturas na tampa;

- É interessante ainda aumentar o número (4 para 12) ou o diâmetro $(25,4 \mathrm{~mm}$ para $76,2 \mathrm{~mm}$ ) dos injetores para tornar o processo de inertização em estudo robusto em relação a uma possível má selagem de aberturas na tampa.

Com base no estudo feito, são dadas as seguintes recomendações para a correta prática de inertização de distribuidores:

- Deve-se atentar para a entrada de ar no distribuidor durante o projeto ou aprimoramento de sistemas de inertização;

- Não se deve posicionar injetores de argônio desprotegidos do ar ambiente;

- A maior vazão disponível nem sempre é suficiente ou a melhor opção;

- É altamente recomendado o uso de medidores de vazão calibrados.

* Contribuição técnica ao 450 Seminário de Aciaria - Internacional, 25 a 28 de maio de 2014, Porto Alegre, RS, Brasil. 


\section{Agradecimentos}

Os autores agradecem a Fapemig pelo apoio financeiro concedido ao projeto Processo No TEC - APQ-00373-11 - "Modelamento físico e matemático do escoamento multifásico em sistemas metalúrgicos"

Os autores agradecem também o apoio da CAPES/PROEX ao programa de pósgraduação PPGEM/UFMG.

O primeiro autor agradece ao CNPq pela concessão da bolsa de mestrado.

\section{REFERÊNCIAS}

1 Zhang L, Thomas BG. State of the art in evaluation and control of steel cleanlinesss. ISIJ International. 2003;43(3):271-291.

2 Bonilla C. Slivers in continuous casting. In: $78^{\text {th }}$ Steelmaking Conference Proceedings; 1995; Nashville, USA. Iron and Steel Society; 1995. p.743-752.

3 Hughes KP, Schade CT, Shepherd MA, Weyant JW. Improvement in the internal quality of continuously cast slabs at Lukens steel. Iron \& Steelmaker. 1995;22(6):3541.

4 Yuan C. Reduction of blister defects in steel plates. SEAISI Quarterly Journal. 2009;38(4):16-19.

5 Buoro,S, Romanelli G. Technological review of the start-up of a new jumbo bloom continuous caster for specialty steels. Iron \& Steel Technology. 2012; jul:39-47.

6 Chen J, Chen CC, Yang CY, Yang CC. Development of inert casting system for slab caster in CSC. In: AISTech, 2005, p. 635-642.

7 Riley MF. Control of Gas Turbulence in Purging of Caster Tundishes. In: $79^{\text {th }}$ Steelmaking Conference Proceedings; 1996; Pittsburgh, USA. Iron and Steel Society; 1996. p.513-518.

8 Mattedi S, Furtado HS, Garcia UM, Guimarães FMQ, Barbabela BA, Rubião LEG. Argon injection optimization at the CST continuous casting machine. In: AISE Annual Convention and Exposition; 2003; Pittsburgh, USA. Association of Iron and Steel Engineers; 2003. p.1-22.

9 Uesugi T. Production of high carbon chromium bearing steel by vertical type continuous caster. Tetsu-to-Hagané. 1985;71(14):1631-38.

10 Bannenberg N, Harste K. Improvements in steel cleanliness by tundish inertisation. La Revue de Métallurgie.1993; 90(1):71-76.

11 Ansys. Ansys Cfx-Solver theory guide, 14.5. Canonsburg: Ansys; 2012.

12 Incropera FP, DeWitt DP, Bergman TL, Lavine AS. Fundamentals of heat and mass transfer. 6 ed. Hoboken: Willey; 2006. p.997.

13 Bich E, Millat J, Vogel E. The viscosity and thermal conductivity of pure monatomic gases from their normal boiling point up to $5000 \mathrm{~K}$ in the limit of zero density and at $0.101325 \mathrm{MPa}$. Journal of Physical and Chemical Reference Data. 1990;19(6):12891305.

14 Atkins P, De Paula J. Atkins' physical chemistry. 8 ed. New York: Oxford University Press; 2006. p.1064.

15 Bird RB, Stewart WE, Lightfoot EN. Transport Phenomena. 2 ed. New York: John Wiley \& Sons; 2002. p.905.

\footnotetext{
* Contribuição técnica ao 450 Seminário de Aciaria - Internacional, 25 a 28 de maio de 2014,
} Porto Alegre, RS, Brasil. 